Pacific

Journal of

Mathematics

COTILTING VERSUS PURE-INJECTIVE MODULES

Francesca Mantese, Alberto Tonolo, and Pavel Ruzicka 


\title{
COTILTING VERSUS PURE-INJECTIVE MODULES
}

\author{
Francesca Mantese, Alberto Tonolo, and Pavel Ruzicka
}

\begin{abstract}
Let $R$ and $S$ be arbitrary associative rings. A left $R$-module ${ }_{R} W$ is said to be cotilting if the class of modules cogenerated by ${ }_{R} W$ coincides with the class of modules for which the functor $\operatorname{Ext}_{R}^{1}(-, W)$ vanishes. In this paper we characterize the cotilting modules which are pure-injective. The two notions seem to be strictly connected: Indeed all the examples of cotilting modules known in the literature are pure-injective. We observe that if ${ }_{R} W_{S}$ is a pure-injective cotilting bimodule, both $R$ and $S$ are semiregular rings and we give a characterization of the reflexive modules in terms of a suitable "linear compactness" notion.
\end{abstract}

\section{Introduction.}

Cotilting modules first appeared as vector space duals of tilting modules over finite dimensional algebras [12, IV, 7.8]. Recently they have been introduced [5] in the framework of modules over arbitrary associative rings, acquiring a proper independent role. The cotilting modules generalize the notion of injective cogenerator: They are injectives with respect to short exact sequences of modules cogenerated by them.

For arbitrary rings $R$ and $S$, a Morita duality between left $R$-modules and right $S$-modules is given by the contravariant Hom functors associated to a Morita bimodule, i.e., a faithfully balanced bimodule ${ }_{R} W_{S}$ with ${ }_{R} W$ and $W_{S}$ both injective cogenerators. One of the major component in the theory of Morita dualities is Müller's theorem [13] which states that the reflexive modules are precisely the linearly compact modules. If ${ }_{R} W_{S}$ is a Morita bimodule, both $R$ and $S$ are semiperfect rings [16, Theorem 2.7].

For arbitrary rings $R$ and $S$, a cotilting duality between left $R$-modules and right $S$-modules is given by the contravariant Hom functors and the contravariant Ext functors associated to a cotilting bimodule, i.e., a faithfully balanced bimodule ${ }_{R} W_{S}$ with both ${ }_{R} W$ and $W_{S}$ cotilting modules (see $[4])$.

All known examples of cotilting modules are pure-injective. In this paper we characterize the pure-injective cotilting modules. We observe that if ${ }_{R} W_{S}$ is a pure-injective cotilting bimodule, both $R$ and $S$ are semiregular 
rings and we give a characterization of the reflexive modules in terms of a suitable "linear compactness" notion.

\section{About the pure-injectivity of a cotilting module.}

Let $R$ be an associative ring with $1 \neq 0$. We denote by $R$-Mod the category of left unitary $R$-modules and their homomorphisms. Given a left $R$-module $W$, we consider the following classes:

- Cogen $W$ denotes the class of all left $R$-modules cogenerated by ${ }_{R} W$, that is all $M$ in $R$-Mod such that there exist a cardinal $\lambda$ and a monomorphism $M \hookrightarrow W^{\lambda}$;

- ${ }^{\perp} W$ denotes the class of all left $R$-modules $M$ such that $\operatorname{Ext}_{R}^{1}(M, W)=$ 0 .

A left $R$-module ${ }_{R} W$ is said to be cotilting [5] if $\operatorname{Cogen}_{R} W={ }^{\perp} W$. The cotilting modules generalize injective cogenerators: Clearly ${ }_{R} W$ is an injective cogenerator if and only if both the classes $\operatorname{Cogen}_{R} W$ and ${ }^{\perp} W$ coincide with the whole category of left $R$-modules. A short exact sequence

$$
0 \rightarrow K \rightarrow L \rightarrow M \rightarrow 0
$$

is said to be pure if any morphism $P \rightarrow M$, with $P$ finitely presented, lifts to a morphism $P \rightarrow L$.

Definition 1.1. A module ${ }_{R} W$ is pure-injective if it is injective with respect to any pure exact sequence.

All known examples of cotilting modules are pure-injective. It naturally arises the question how the two notions are related.

Proposition 1.2. Let ${ }_{R} W$ be a cotilting module. If the class Cogen $W$ is closed under direct limits, then $W$ is pure-injective.

Proof. Let us show that for any pure exact sequence $0 \rightarrow A \rightarrow B \rightarrow C \rightarrow 0$ and for any map $f: A \rightarrow W$ there exists a map $g$ making the following diagram commute:

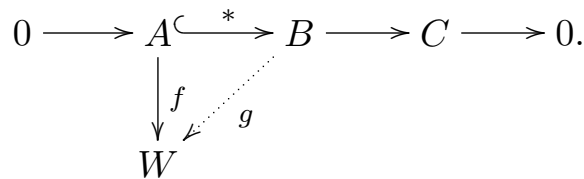

Replacing $A \stackrel{*}{\hookrightarrow} B$ by $A / \operatorname{Rej}_{W} A \stackrel{*}{\hookrightarrow} B / \operatorname{Rej}_{W} A$,we can assume that $\operatorname{Rej}_{W} A$ $=0$. The pure exact sequence $0 \rightarrow A \rightarrow B \rightarrow C \rightarrow 0$ is a direct limit of split exact sequences $0 \rightarrow A \rightarrow B_{i} \rightarrow C_{i} \rightarrow 0$ with $C_{i}$ finitely presented (cf. 34.2 [14]). For each index $i$ we have the commutative diagram with exact rows 
and columns:

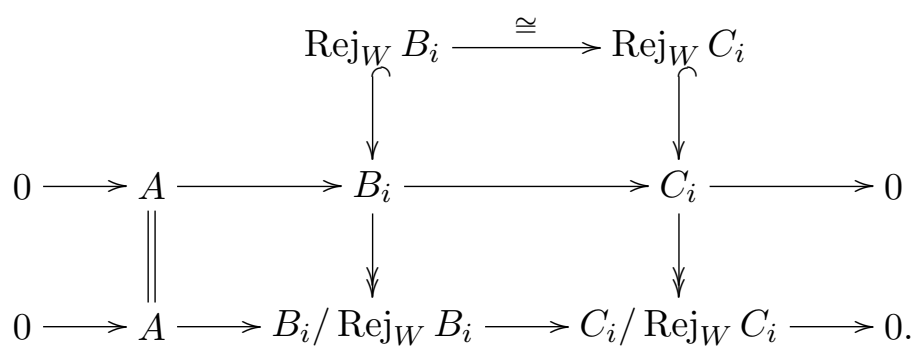

Applying the direct limit functor we get

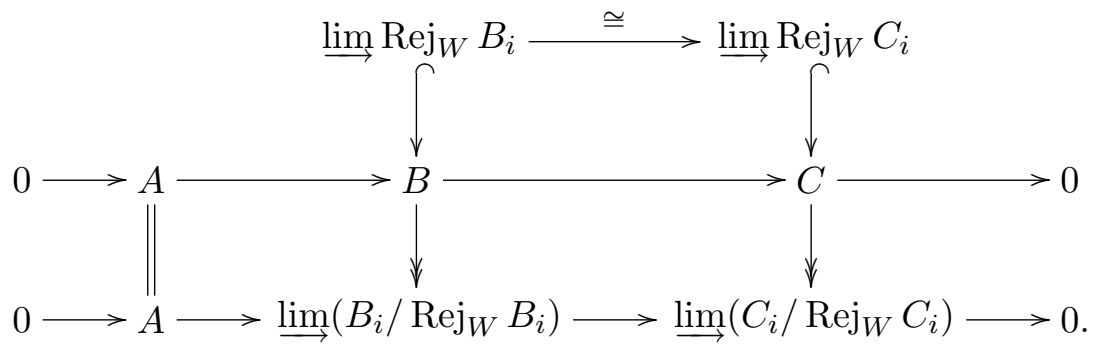

Since $\lim _{\longrightarrow} \operatorname{Rej}_{W} B_{i}$ is in the kernel of $\operatorname{Hom}(-, W)$ and $\lim _{\longrightarrow}\left(B_{i} / \operatorname{Rej}_{W} B_{i}\right)$ belongs to Cogen $W$ by the assumption, we infer that $\underset{\lim }{\longrightarrow}\left(B_{i} / \operatorname{Rej}_{W} B_{i}\right) \cong$ $B / \operatorname{Rej}_{W} B$. Also $\underset{\longrightarrow}{\longrightarrow}\left(C_{i} / \operatorname{Rej}_{W} C_{i}\right)$ belongs to Cogen $W={ }^{\perp} W$. So $f$ can be extended to a morphism $g^{\prime}: B / \operatorname{Rej}_{W} B \rightarrow W$; the composition of $g^{\prime}$ with the canonical projection $B \rightarrow B / \operatorname{Rej}_{W} B$ yields the desired map $g$.

In the cotilting case, since Cogen $W={ }^{\perp} W$, the hypothesized closure under direct limits of the class of modules cogenerated by $W$ is suggested by the following proposition:

Proposition 1.3. If ${ }_{R} W$ is a pure-injective module, then $\stackrel{\perp}{R} W$ is closed under direct limits.

Proof. Consider a direct system $\left\{M_{i}: i \in I\right\}$ in ${ }^{\perp} W$. The canonical exact sequence

$$
0 \rightarrow K \rightarrow \oplus_{i \in I} M_{i} \rightarrow \underline{\lim }_{i \in I} M_{i} \rightarrow 0
$$

is pure $(\mathrm{cf} .[\mathbf{1 4}, 33.9,(2)])$. Applying $\operatorname{Hom}_{R}(-, W)$ we get the long exact sequence

$$
\begin{aligned}
\quad \cdots \rightarrow \operatorname{Hom}\left(\oplus_{i \in I} M_{i}, W\right) \stackrel{f}{\rightarrow} \operatorname{Hom}(K, W) \rightarrow \operatorname{Ext}_{R}^{1}\left(\stackrel{\lim }{\longrightarrow}_{i \in I} M_{i}, W\right) \rightarrow & \\
& \rightarrow \operatorname{Ext}_{R}^{1}\left(\oplus_{i \in I} M_{i}, W\right)=0 .
\end{aligned}
$$

Since $W$ is pure-injective, $f$ is surjective; so $\lim _{i \in I} M_{i}$ belongs to ${ }^{\perp} W$.

This result has been used in [9, Lemma 9] to prove that, if $\mathcal{C}$ is a class of pure-injective modules, every module $M$ which has a ${ }^{\perp} \mathcal{C}$-precover has a 
${ }^{\perp} \mathcal{C}$-cover (see [15] for an extensive introduction to theory of (pre)covers and (pre)envelopes of modules, including various recent results).

Corollary 1.4. If ${ }_{R} W$ is a cotilting module, then $W$ is pure-injective if and only if Cogen $W$ is closed under direct limits. In such a case any module has a Cogen $W$-cover.

Proof. The first claim follows by Propositions 1.2 and 1.3. The second one follows by [1, Corollary 2.6].

Open Problem 1.5. Are all cotilting (bi)modules pure-injective? ${ }^{1}$

It is well-known that the endomorphism rings of a Morita bimodule are semiperfect; indeed a Morita bimodule is injective and finitely cogenerated on both sides (see [16, Theorem 2.7, Proposition 1.19]). We are able to give an analogous result for cotilting bimodules, assuming the closure under direct limits of the classes cogenerated by them. We recall that a ring $R$ is said to be semiregular if $R / J(R)$ is regular and the idempotents lift over the Jacobson radical $J(R)$.

Proposition 1.6. Let ${ }_{R} W_{S}$ be a pure-injective cotilting bimodule, i.e., faithfully balanced and cotilting and pure-injective on both sides. Then both $R$ and $S$ are semiregular rings.

Proof. The notions of pure-injective and algebraically compact module coincide (cf. $[\mathbf{1 4}, 34.4])$. Then, by $[\mathbf{1 7}$, Theorem 9], $R$ and $S$ are both semiregular.

Remark 1.7. Observe that if the ring $R$ is regular, the classes of pureinjective and of injective $R$-modules coincide $[14,37.6]$. Therefore pureinjective cotilting bimodules which are not Morita bimodules "live in the space between semiregular and regular rings".

\section{Characterizing the reflexive modules.}

Let ${ }_{R} W_{S}$ be an arbitrary bimodule. In the sequel we denote by $\Delta$ the functors $\operatorname{Hom}_{?}(-, W)$, and by $\Gamma$ the functors $\operatorname{Ext}_{?}^{1}(-, W)$, where ? stands for $R$ or $S$. We denote by $\Delta^{2}$ both the compositions $\operatorname{Hom}_{R}\left(\operatorname{Hom}_{S}(-, W), W\right)$ and $\operatorname{Hom}_{S}\left(\operatorname{Hom}_{R}(-, W), W\right)$. Given a (left $R$, or right $S$ )-module $M$, we denote by $\delta_{M}$ the canonical homomorphism $M \rightarrow \Delta^{2}(M)$ defined by $m \mapsto$ $[f \mapsto f(m)]$. A module $M$ is called reflexive (resp. torsionless) if $\delta_{M}$ is an isomorphism (resp. monomorphism).

Clearly a torsionless module $M$ is reflexive if and only if the evaluation map $\delta_{M}$ is surjective. Endowed $M$ and $\Delta^{2} M$ with any topology, the surjectivity of $\delta_{M}$ can be tested in a topological way asking for $\operatorname{Im} \delta_{M}$ to be both dense and closed in $\Delta^{2} M$. As the approach of Müller [13] to the classical

\footnotetext{
${ }^{1}$ Recently Silvana Bazzoni proved that any cotilting module is pure-injective [2].
} 
case of Morita dualities suggests, we introduce topological tools in order to characterize the reflexive modules.

Let us endow $\Delta^{2} M$ with the finite topology $\varphi$ : The linear topology for which the family of submodules $V(F)=\left\{\alpha \in \Delta^{2} M: \alpha(F)=0\right\}$, where $F$ is a finite subset of $\Delta M$, is a base for the filter of neighbourhoods of zero.

Let us endow $W$ with the discrete topology. Given any torsionless module $M$, we associate with each subset $A$ of $\Delta M$ the weak topology with respect to morphisms in $A$, denoted by $\tau_{A}$. By definition $\tau_{A}$ is the coarsest topology on $M$ such that all morphisms in $A$ are continuous: It is a linear topology with a base for its filter of neighbourhoods of zero formed by finite intersections of kernels of morphisms in $A$. In the sequel the topology $\tau_{\Delta M}$ will be shortly denoted by $\tau$. Note that $\tau$ is the maximum element of the set of linear topologies $\left\{\tau_{A}: A \subseteq \Delta M\right\}$ partially ordered by inclusion. Let $L_{\sigma}$ be a linearly topologized module. Denote by $H$ the $\sigma$-closure of zero in $L$. Note that $H$ is equal to the intersection of all neighbourhoods of zero and, since $\sigma$ is a linear topology, $\sigma$ is Hausdorff if and only if $H=0$. A $\sigma$-Cauchy net in $L$ is a family $X_{\lambda}, \lambda \in \Lambda$, indexed by the upwards directed partially ordered set $\Lambda$, such that for every neighbourhood $U$ of zero there exists an upper subset $\Lambda^{\prime}$ of $\Lambda$ with $x_{\lambda}-x_{\lambda^{\prime}} \in U$ for every $\lambda, \lambda^{\prime} \in \Lambda^{\prime}$. The topology $\sigma$ is complete, i.e., any $\sigma$-Cauchy net in $L$ converges in $L$, if and only if the topological quotient $L / H$ is complete (see [3, Chap. $3, \S 2]$ ). Note that a closed submodule of a complete module is complete. The completion of $L / H$ is called the Hausdorff completion of $L$ : Denoted by $\mathcal{J}=\left\{J_{\lambda}: \lambda \in \Lambda\right\}$ a base for the filter of neighbourhoods of zero in $L_{\sigma}$ consisting of open submodules, it coincides with the inverse $\operatorname{limit} \varliminf_{L} L / J_{\lambda}$ (see [10, Proposition 13.7]).

Proposition 2.1. Let $M$ be a torsionless module.

(i) The topologies $\tau$ on $M$ and $\varphi$ on $\Delta^{2} M$ are Hausdorff.

(ii) $\delta_{M}: M_{\tau} \rightarrow \Delta^{2} M_{\varphi}$ is a topological embedding.

(iii) The topology $\varphi$ on $\Delta^{2} M$ is complete.

Proof. (i) Since $M$ is cogenerated by $W$, there exists a set $X$ and the following maps:

$$
M \stackrel{i}{\hookrightarrow} W^{X} \stackrel{\pi_{x}}{\longrightarrow} W .
$$

Clearly $\{0\}$ is the intersection of $\operatorname{Ker}\left(\pi_{x} \circ i\right), x \in X$. Since any $\operatorname{Ker}\left(\pi_{x} \circ i\right)$ is $\tau$-open and hence $\tau$-closed, $\tau$ is Hausdorff. Let us consider the topology $\varphi$. The open submodules $V(F)=\left\{\alpha \in \Delta^{2} M: \alpha(F)=0\right\}$, with $F$ finite subset of $\Delta M$, have intersection zero: Hence $\varphi$ is Hausdorff.

(ii) It follows from the fact that for any finite subset $F$ of $\Delta M$ we have

$$
V(F) \cap \delta_{M}(M)=\left\{\delta_{M}(m): f(m)=0 \forall f \in F\right\}=\delta_{M}\left(\cap_{f \in F} \operatorname{Ker} f\right) .
$$

(iii) The topological module $\Delta^{2} M_{\varphi}$ is a closed submodule of $W^{\Delta M}$ endowed with the product of the discrete topologies. Since the product of complete topologies is also complete, we can conclude. 
Corollary 2.2. Each reflexive module $M$ endowed with the topology $\tau$ is complete.

Proof. If $M$ is reflexive, $\delta_{M}$ is an isomorphism. Therefore, by Proposition 2.1, (ii), it is a topological isomorphism. We conclude by Proposition 2.1, (iii).

Proposition 2.3. For a torsionless module $M$ the following statements are equivalent:

(i) $M$ is reflexive.

(ii) $\tau$ is a complete topology and $\delta_{M}(M)$ is dense in $\Delta^{2} M_{\varphi}$.

Proof. (i) $\Rightarrow$ (ii): It follows by Corollary 2.2 .

(ii) $\Rightarrow$ (i): Since $\tau$ is complete, by Proposition $2.1 \delta_{M}(M)$ is a complete, and hence closed, topological submodule of $\Delta^{2} M_{\varphi}$. Being $\delta_{M}(M)$ dense in $\Delta^{2} M_{\varphi}$ and $\varphi$ an Hausdorff topology, $\delta_{M}$ is an isomorphism.

As suggested by Müller [13] in the case of Morita dualities, we look for a suitable notion of compactness for a module $M$ in order to guarantee the completeness of the topology $\tau$ on $M$.

Note that $\delta_{M}(M)$ is dense in $\Delta^{2} M_{\varphi}$ if and only if for each $\alpha$ in $\Delta^{2} M$ and $f_{1}, \ldots, f_{n}$ in $\Delta M$ there exists $m$ in $M$ such that $\alpha\left(f_{i}\right)=f_{i}(m)$ for each $i=1, \ldots, n$. Following [11], a module $M$ satisfying the above property will be called $W$-dense.

Definition 2.4. Let $M$ be a torsionless left $R$-module. A submodule $K$ of $M$ is called $W$-closed if $M / K$ is torsionless (see [11, $\S 2]$ ). A linear topology on $M$ is said to be a $W$-topology if it has a basis of neighbourhoods of zero consisting of $W$-closed submodules.

Each $W$-closed submodule $K$ of $M$ is closed in $M_{\tau}$. Indeed, for a suitable set $X$, there exist the following maps:

$$
M \stackrel{\pi}{\rightarrow} M / K \stackrel{i}{\hookrightarrow} W^{X} \stackrel{\pi_{x}}{\rightarrow} W .
$$

Then, $K=\bigcap_{x \in X} \operatorname{Ker}\left(\pi_{x} \circ i \circ \pi\right)$ is closed, since it is an intersection of open and hence closed submodules. The converse is not true in general.

Example 2.5. Let $R$ denote the $k$-algebra given by the quiver $1 \rightarrow 2 \rightarrow 3$. It is easy to verify that ${ }_{R} R_{R}$ is a cotilting bimodule. Consider the projective $R$-module $P(2)$. The topology $\tau_{\Delta P(2)}$ is discrete since $P(2)$ embeds in ${ }_{R} R$. Therefore each submodule of $P(2)$, in particular the simple module $S(3)$, is closed. Nevertheless $S(3)$ is not a $R$-closed submodule of $P(2)$, since $P(2) / S(3) \cong S(2)$ is not cogenerated by ${ }_{R} R$.

Definition 2.6. A left $R$-module $M$ is said to be $W$-linearly compact (see $[11, \S 3]$ ), briefly $W$-lc, (resp. $H W$-linearly compact, briefly $H W$-lc) if it is complete in any $W$-topology (resp. in any Hausdorff $W$-topology). 
Analogously to the usual linear compactness, a left $R$-module $M$ is $W$ linear compact if and only if any finitely solvable system of congruences $x \equiv x_{\lambda} \bmod M_{\lambda}$, where $\left\{M_{\lambda}: \lambda \in \Lambda\right\}$ is a downwards directed collection of $W$-closed submodules of $M$, is solvable. Similarly a module $M$ is $H W$ linearly compact if and only if it satisfies the previous condition restricted to downwards directed collections of $W$-closed submodules of $M$ with intersection equal to zero.

Proposition 2.7. Let ${ }_{R} M$ be a torsionless left $R$-module. If $M$ is $H W$ linearly compact, then $M$ is complete in the topology $\tau$.

Proof. Since the intersection of the kernels of a finite number of elements of $\Delta M$ is a $W$-closed submodule of $M, \tau$ is a $W$-topology. Since $M$ is torsionless, by Proposition 2.1 the topology $\tau$ is Hausdorff. Since $M$ is $H W$-lc, $\tau$ is complete.

Corollary 2.8. Let ${ }_{R} M$ be a torsionless left $R$-module. If $M$ is $H W$ linearly compact and $W$-dense, then $M$ is reflexive.

Proof. It follows by Propositions 2.3 and 2.7 .

We can obtain a more precise result for cotilting bimodules.

Theorem 2.9. Let ${ }_{R} W_{S}$ be a cotilting bimodule. For a torsionless left $R$ module $M$ the following statements are equivalent:

(a) $M$ is $H W$-linearly compact and $W$-dense.

(b) $M$ is reflexive and $\tau$ is the unique Hausdorff topology among those induced by subsets of $\Delta M$.

Proof. $(\mathrm{a} \Rightarrow \mathrm{b})$ : By Corollary 2.8 we only have to prove that $\tau$ is the unique Hausdorff topology induced by subsets of $\Delta M$. Since $\tau$ is the maximum element in $\left\{\tau_{A}: A \subseteq \Delta M\right\}$, it is sufficient to prove that if $\tau_{A}$ is Hausdorff, then $\tau$ is coarser than $\tau_{A}$ and hence $\tau_{A}=\tau$.

Let $F$ be a finite subset of $A$. We denote by $f_{F}: M \rightarrow W^{F}$ the diagonal morphism. Let $M_{F}:=\bigcap_{f \in F} \operatorname{Ker} f=\operatorname{Ker} f_{F}$ and $N_{F}:=M / M_{F}$. By [4, Proposition 5] both the left $R$-modules $M_{F}$ and $N_{F}$ are reflexive. We call $\pi_{F}$ the induced map $M \rightarrow N_{F}$. Since $\left\{M_{F}: F \subseteq A, F\right.$ finite $\}$ is a base for the filter of $\tau_{A}$-neighbourhoods of zero consisting of open submodules, $\lim _{F} N_{F}$ is the Hausdorff completion of $M$ endowed with the topology $\tau_{A}$.

But, since $\tau_{A}$ is an Hausdorff $W$-topology and, by hypothesis, $M$ is $H W$ lc, $\tau_{A}$ is complete. Thus $M \cong \lim N_{F}$.

Applying the functors $\Delta$ and lim to the exact sequences

$$
0 \rightarrow M_{F} \rightarrow M \stackrel{\pi_{F}}{\rightarrow} N_{F} \rightarrow 0
$$

we get the exact sequence of right $S$-modules

$$
0 \longrightarrow \lim _{\longrightarrow} \Delta N_{F} \stackrel{\lim \Delta\left(\pi_{F}\right)}{\longrightarrow} \Delta M \longrightarrow \underline{\lim } \Delta M_{F} \longrightarrow 0
$$


Now $\Delta\left(\lim _{\longrightarrow} \Delta\left(\pi_{F}\right)\right) \cong \lim ^{2} \Delta^{2}\left(\pi_{F}\right) \cong \lim _{F}$ is an isomorphism. Then, from the exact sequence

$$
0 \rightarrow \Delta \underline{\lim } \Delta M_{F} \longrightarrow \Delta^{2} M \stackrel{\Delta\left(\lim _{\longrightarrow} \Delta\left(\pi_{F}\right)\right)}{\longrightarrow} \Delta \stackrel{\lim }{\longrightarrow} \Delta N_{F} \longrightarrow \Gamma \stackrel{\lim }{\longrightarrow} \Delta M_{F} \rightarrow 0,
$$

we get $\underline{\lim } \Delta M_{F}$ belongs to Ker $\Delta \cap \operatorname{Ker} \Gamma=0$. Hence $\Delta M \cong \lim \Delta N_{F}$.

Let now $g$ be in $\Delta M$. Since $g$ belongs to $\Delta\left(\pi_{F}\right)\left(\Delta\left(N_{F}\right)\right)$ for some finite subset $F$ of $A$, there exists a morphism $h: N_{F} \rightarrow W$ such that $g=h \circ \pi_{F}$. Then since $\operatorname{Ker} g \supseteq \operatorname{Ker} \pi_{F}=\bigcap_{f \in F} \operatorname{Ker} f$, Ker $g$ is $\tau_{A}$-open. Therefore $\tau$ is coarser than $\tau_{A}$.

( $b \Rightarrow$ a): We only have to prove that $M$ is $H W$-lc. Let $\sigma$ be a Hausdorff $W$-topology on $M$. By definition $\sigma$ has a basis $\mathcal{B}$ for the filter of neighbourhoods of zero consisting of $W$-closed submodules; since $\sigma$ is Hausdorff, the intersection of elements in $\mathcal{B}$ is equal to zero. Observe that any element $V$ of $\mathcal{B}$ is the intersection of the kernels of a (not necessarily finite) subset $A_{V}$ of $\Delta M$. Let $A$ the union $\cup_{V \in \mathcal{B}} A_{V}$. If $f$ belongs to $A_{V}$, Ker $f$ contains $V$ and hence it is $\sigma$-open. Therefore the topology $\tau_{A}$ is coarser than $\sigma$. Since

$$
\cap_{f \in A} \operatorname{Ker} f=\cap_{V \in \mathcal{B}} V=\{0\},
$$

0 is a closed subset of $M_{\tau_{A}}$, i.e., $\tau_{A}$ is Hausdorff. By hypothesis $\tau_{A}=\tau$ and, since $M$ is reflexive, by Proposition $2.3 \tau_{A}$ is complete. By [3, Proposition III.3.10], also the topology $\sigma$ is complete.

Lemma 2.10. Let ${ }_{R} W_{S}$ be a cotilting bimodule with Cogen $W_{S}$ closed under direct limits. Let $M$ be a reflexive left $R$-module. Then $\tau$ is the unique Hausdorff topology among those induced by subsets of $\Delta M$.

Proof. We can follow the first two paragraphs of the proof of Theorem 2.9, $(\mathrm{a} \Rightarrow \mathrm{b})$. Applying the functors $\Delta$ and $\underline{\mathrm{lim}}$ to the exact sequence

$$
0 \rightarrow M_{F} \rightarrow M \stackrel{\pi_{F}}{\rightarrow} N_{F} \rightarrow 0
$$

we get the exact sequence

$$
0 \rightarrow \underline{\lim } \Delta N_{F} \stackrel{\lim _{\longrightarrow} \Delta\left(\pi_{F}\right)}{\longrightarrow} \Delta M \longrightarrow \lim _{\longrightarrow} \Delta M_{F} \rightarrow 0
$$

of right $S$-modules. Observe that $\lim _{\longrightarrow} \Delta M_{F}$ again belongs to Ker $\Gamma=$ Cogen $W$ since, by hypothesis, Cogen $\vec{W}$ is closed under direct limits. Moreover

$$
\Delta \lim _{\longrightarrow} \Delta M_{F} \cong \lim _{\longleftarrow} \Delta^{2} M_{F} \cong \lim _{\longleftarrow} M_{F}=\cap_{f \in A} \operatorname{Ker} f=0 .
$$

Therefore, since Ker $\Delta \cap \operatorname{Ker} \Gamma=0$, we get $\lim _{\longrightarrow} \Delta M_{F}=0$. Hence $\Delta M \cong$ $\underset{\lim }{\longrightarrow} \Delta N_{F}$. We can thus conclude following the last paragraph of the proof

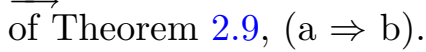

Thus we obtain the characterization of reflexive modules for pure-injective cotilting bimodules. 
Theorem 2.11. Let ${ }_{R} W_{S}$ be a pure-injective cotilting bimodule. For a torsionless module $M$ the following are equivalent:

(a) $M$ is reflexive.

(b) $M$ is $H W$-linearly compact and $W$-dense.

(c) $M$ is $W$-linearly compact and $W$-dense.

Proof. $(\mathrm{c} \Rightarrow \mathrm{b} \Rightarrow \mathrm{a})$ : They follow by Definition 2.6 and by Theorem 2.9 .

$(\mathrm{a} \Rightarrow \mathrm{c})$ : Trivially $M$ is $W$-dense. Let $\sigma$ be a $W$-topology on $M$ and let $H \leq M$ be the $\tau$-closure of zero. Since $H$ is a $W$-closed submodule of $M$, $M / H$ is reflexive (see [4, Proposition 5]). By Theorem 2.9 and Lemma 2.10 $M / H$ is $H W$-lc and hence complete endowed with the quotient topology of $\tau$. Therefore $M_{\tau}$ is complete.

In [13] Müller proved that if ${ }_{R} W_{S}$ is a Morita bimodule, a module $M$ is reflexive if and only if it is linearly compact in the discrete topology if and only if it is complete in any Hausdorff linear topology. In such a case $W$ cogenerates the whole category of modules, hence any submodule is $W$-closed. Therefore the notions of $W$-linear compactness, of $H W$-linear compactness and of linear compactness in the discrete topology coincide. In our setting a density condition comes out. Let us better investigate its role.

Proposition 2.12. Let ${ }_{R} W_{S}$ be a bimodule such that Cogen $W_{S} \subseteq \operatorname{Ker} \Gamma$. A left $R$-module $M$ is $W$-dense if and only if $\operatorname{Im}(f)$ is a reflexive left $R$-module for every $f \in \operatorname{Hom}_{R}\left(M, W^{n}\right),(n \in \mathbb{N})$.

Proof. Let us consider for each $f$ in $\operatorname{Hom}\left(M, W^{n}\right), n \in \mathbb{N}$, the following commutative diagram of linearly topologized modules and continuous morphisms:

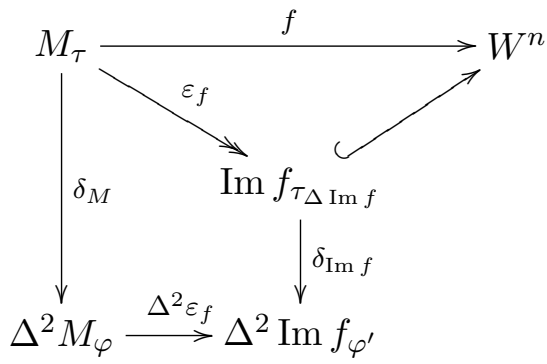

where $\varphi^{\prime}$ is the finite topology on $\Delta^{2} \operatorname{Im} f$. Since $\operatorname{Im} f \leq W^{n}, \tau_{\Delta \operatorname{Im} f}$ is the discrete topology; in particular it is complete.

Suppose that $M$ is $W$-dense. Applying $\Delta$ to the exact sequence $0 \rightarrow$ Ker $f \rightarrow M \stackrel{\varepsilon_{f}}{\rightarrow} \operatorname{Im} f \rightarrow 0$, we get the exact sequence $0 \rightarrow \Delta \operatorname{Im} f \stackrel{\Delta\left(\varepsilon_{f}\right)}{\longrightarrow}$ $\Delta M \rightarrow C \rightarrow 0$ with $C$ in Cogen $W_{S}$. Since Cogen $W_{S} \subseteq \operatorname{Ker} \Gamma, \Delta^{2}\left(\varepsilon_{f}\right)$ is an epimorphism. Therefore, the $W$-density of $M$ implies the $W$-density of $\operatorname{Im} f$. By Proposition 2.3, $\operatorname{Im} f$ is reflexive. 
Conversely, let $f_{1}, \ldots, f_{n}$ be in $\Delta M$. We denote by $f: M \rightarrow W^{n}$ their diagonal morphism, $m \mapsto\left(f_{1}(m), \ldots, f_{n}(m)\right)$, and by $\mu_{f} \circ \varepsilon_{f}$ the usual factorization of $f$ through $\operatorname{Im} f$. Since $\operatorname{Im} f$ is reflexive, for each $\alpha$ in $\Delta^{2} M$ there exists $m_{\alpha}$ in $M$ such that

$$
\Delta^{2}\left(\varepsilon_{f}\right)(\alpha)=\delta_{\operatorname{Im} f}\left(f\left(m_{\alpha}\right)\right) .
$$

In particular, denoted by $p_{i}: \operatorname{Im} f \rightarrow W$ the $i$-th projection, we have

$$
\begin{gathered}
\left(\Delta^{2}\left(\varepsilon_{f}\right)(\alpha)\right)\left(p_{i}\right)=\alpha\left(\Delta\left(\varepsilon_{f}\right)\right)\left(p_{i}\right)=\alpha\left(p_{i} \circ \varepsilon_{f}\right)=\alpha\left(f_{i}\right) \\
\left(\delta_{\operatorname{Im} f}\left(f\left(m_{\alpha}\right)\right)\left(p_{i}\right)=p_{i}\left(f\left(m_{\alpha}\right)\right)=f_{i}\left(m_{\alpha}\right) ;\right.
\end{gathered}
$$

therefore $\alpha$ and $\delta_{M}\left(m_{\alpha}\right)$ coincide on $f_{1}, \ldots, f_{n}$. Therefore $M$ is $W$-dense.

If ${ }_{R} W_{S}$ is a Morita bimodule, then the class of reflexive modules contains $W$ and it is closed under submodules and finite direct sums. Therefore the $W$-density condition is always satisfied: Any module $M$ is $W$-dense. This is not the case for cotilting bimodules.

Example 2.13. Let $k$ be an algebraically closed field. Denote by $A$ the generalized Kronecker algebra of dimension $\aleph_{0}$ over $k$ given by the quiver

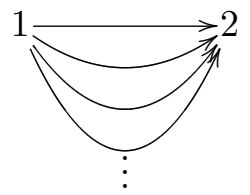

with a countable set of arrows from 1 to 2 , i.e., the ring of lower triangular matrices

$$
\left(\begin{array}{cc}
k & 0 \\
V & k
\end{array}\right)=\left\{\left(\begin{array}{cc}
a & 0 \\
v & b
\end{array}\right): a, b \in k, v \in V\right\}
$$

where $V$ is a $k$-vector space of dimension $\aleph_{0}$ (see $[\mathbf{7}, \mathbf{8}]$ ). Then, by $[\mathbf{8}$, Lemma 2.2], $A$ is a hereditary, coherent and perfect ring. It is easily verified that ${ }_{A} A_{A}$ is a cotilting bimodule and $\operatorname{Cogen}(A)$ consists of projective modules, while $\operatorname{Ker}(\Delta)$ contains exactly the modules without projective direct summands. The reflexive modules coincide with the finitely generated projective modules [8, Lemma 2.3]. Denote by $e_{1}, e_{2}$ the primitive idempotents, i.e., $e_{1}=\left(\begin{array}{ll}1 & 0 \\ 0 & 0\end{array}\right), e_{2}=\left(\begin{array}{ll}0 & 0 \\ 0 & 1\end{array}\right)$. Let $P_{i}=A e_{i}$ and $Q_{i}=e_{i} A, i=1,2$. The socle $S$ of $P_{1}$ is isomorphic to $P_{2}^{\left(\aleph_{0}\right)}$; therefore it is a not reflexive submodule of $A$. By Proposition 2.12, $S$ is not $A$-dense.

In [6, Definition 1.2] Colpi and Fuller introduced the $W$-torsionless linear compactness, a generalization of the notion of linear compactness with respect to the torsion theories associated to a cotilting bimodule ${ }_{R} W_{S}$. They prove that if a module is $W$-torsionless linearly compact, then it is reflexive, i.e., (see Proposition 2.3) $\tau$ is a complete topology and $M$ is $W$-dense. 
Our notion of $W$-linear compactness is strong enough to assure the completeness, but to obtain the $\Delta$-reflexivity we need to assume explicitly the $W$-density. Assuming ${ }_{R} W$ and $W_{S}$ pure-injective (as in all examples known in the literature), these two notions together completely characterize the classes of reflexive modules. The notion of $W$-torsionless linear compactness is too strong to characterize the classes of reflexive modules in the general case; this happens if and only if the classes of reflexive left $R$ - and right $S$ - modules are closed under submodules [6, Corollary 1.9]. Observe that in this case, by Proposition 2.12, any module is $W$-dense. Adding the hypotheses of both the contexts we get:

Corollary 2.14. Let ${ }_{R} W_{S}$ be a pure-injective cotilting bimodule. Assume the classes of reflexive left $R$ - and right $S$-modules being closed under submodules. For a module $M$, the following statements are equivalent:

(a) $M$ is reflexive;

(b) $M$ is $W$-torsionless linearly compact;

(c) $M$ is $W$-linearly compact.

Acknowledgement. The results of the first section have been obtained during an informal meeting at the University of Munchen with Lidia Angeleri Hügel and Jan Trlifaj. We wish to thank these colleagues and friends for allowing us to publish in this paper the outcome of those useful discussions. We wish also to thank Riccardo Colpi for his valuable suggestions which improved the paper.

\section{References}

[1] L. Angeleri Hügel, A. Tonolo and J. Trlifaj, Tilting preenvelopes and cotilting precovers, Algebr. Represent. Theory, 4 (2001), 155-170, MR 2002e:16010, Zbl 0999.16007.

[2] S. Bazzoni, Cotilting modules are pure-injective, to appear in Proc. Amer. Math. Soc., 131 (2003), 3665-3672.

[3] N. Bourbaki, Éléments de Mathématique, Topologie Générale, Masson, 1990, MR 50 \#11111, Zbl 0249.54001.

[4] R. Colpi, Cotilting bimodules and their dualities, Proc. Euroconf. Murcia '98, LNPAM, 210, Dekker, New York, 2000, 81-93, MR 2001f:16015, Zbl 0976.16009.

[5] R. Colpi, G. D'Este and A. Tonolo, Quasi-tilting modules and counter equivalences, J. Algebra, 191 (1997), 461-494, MR 99g:16008, Zbl 0876.16004.

[6] R. Colpi and K.R. Fuller, Cotilting modules and bimodules, Pacific J. Math., 192(2) (2000), 275-291, MR 2001f:16014, Zbl 1014.16008.

[7] G. D'Este, Free modules obtained by means of infinite direct products, Proc. International Conference Algebra and its Applications, Contemporary Mathematics, 259 (2000), 161-173, MR 2001g:16006, Zbl 0986.16001. 
[8] _ Reflexive modules are not closed under submodules, Proc. Conference on Representations of Algebras São Paulo '99, Lecture Notes in Pure and Appl. Math., 224, Dekker, New York, 2002, 53-64, CMP 1884 806, Zbl 1013.16002.

[9] P. Eklof and J. Trlifaj, Covers induced by Ext, J. Algebra, 231(2) (2000), 640-651, MR 2001f:16021, Zbl 0981.16010.

[10] L. Fuchs, Infinite Abelian Groups, Vol. 1, Academic Press, New York, 1970, MR 41 \#333, Zbl 0209.05503.

[11] J.L. Gomez Pardo, Counterinjective modules and duality, J. Pure Applied Algebra, 61 (1989), 165-179, MR 90k:16026, Zbl 0687.16021.

[12] D. Happel, Triangulated Categories in the Representation Theory of Finite Dimensional Algebras, Cambridge Univ. Press, Cambridge, 1988, MR 89e:16035, Zbl 0635.16017.

[13] B.J. Müller, Linear compactness and Morita duality, J. Algebra, 16 (1970), 60-66, MR 41 \#8474, Zbl 0206.04803.

[14] R. Wisbauer, Foundations of Module and Ring Theory, Gordon and Breach Science Pulishers, 1991, MR 92i:16001, Zbl 0746.16001.

[15] J. Xu, Flat Covers of Modules, Lecture Notes in Mathematics, 1634, Springer, New York, 1996, MR 98b:16003, Zbl 0860.16002.

[16] W. Xue, Rings with Morita dualities, Lecture Notes in Mathematics, 1523, Springer, New York, 1992, MR 94b:16002, Zbl 0790.16009.

[17] B. Zimmermann Huisgen and W. Zimmermann, Algebraically compact rings and modules, Math. Z., 161 (1978), 81-93, MR 58 \#16792, Zbl 0363.16017.

Received January 8, 2001 and revised January 7, 2002.

Dipartimento di Matematica Pura ed Applicata

UNIVERSITÀ DI PADOVA

VIA BELZONi 7

I-35131 PADOVA

ITALY

E-mail address: fmantese@math.unipd.it

Dipartimento di Matematica Pura ed Applicata

UNIVERSitÀ DI PADOVA

VIA BELZONi 7

I-35131 PADOVA

ITALY

E-mail address: tonolo@math.unipd.it

KATEDRA ALGEBRY MFF UK

SOKOLOVSKÁ 83

18675 Prague 8

Czech Republic

E-mail address: ruzicka@karlin.mff.cuni.cz 